(C) 2020 IEEE

\title{
Inductive Power Transfer System with Series Connected Primary and Independent Secondary Coils
}

\author{
X. Du and D. Dujic
}

This material is posted here with permission of the IEEE. Such permission of the IEEE does not in any way imply IEEE endorsement of any of EPFL's products or services. Internal or personal use of this material is permitted. However, permission to reprint / republish this material for advertising or promotional purposes or for creating new collective works for resale or redistribution must be obtained from the IEEE by writing to pubs-permissions@ieee. org. By choosing to view this document, you agree to all provisions of the copyright laws protecting it. 


\title{
Inductive Power Transfer System with Series Connected Primary and Independent Secondary Coils
}

\author{
Xiaotong Du, Dražen Dujić \\ Power Electronics Laboratory - PEL \\ École Polytechnique Fédérale de Lausanne - EPFL \\ CH-1015 Lausanne, Switzerland \\ xiaotong.du@epfl.ch; drazen.dujic@epfl.ch
}

\begin{abstract}
In this paper, the adoption of the inductive power transfer technique is proposed to achieve contactless auxiliary power supply for power electronics building blocks of medium voltage converters. Due to the high degrees of design freedom and multiple parameters in the system with series connected transmitters, the optimization approach is proposed to realize high efficiency, constant output voltage and low reactive power, simultaneously. In addition, influence of various design parameters on the final design is explored. Finally, the analytical model and design procedure is verified through simulations.
\end{abstract}

Index Terms-Auxiliary power supply, inductive power transfer, compensation networks

\section{INTRODUCTION}

Power electronics building block (PEBB) as the fundamental subassembly in modular converters is widely used in medium voltage applications. Stacked PEBBs in many topologies are normally floating with respect to the ground potential, and there are huge potential differences between reference potential of PEBBs and the reference ground. High insulation capability is required in auxiliary power supply (APS) system [1], [2]. The wireless inductive power (IPT) supply without physical contact between the primary and secondary sides is suitable for this application [3], despite multiple design challenges that must be overcome.

Work presented in [2] uses one transmitter and one receiver coil to supply all stacked PEBBs. However the air transformer is bulky and could not be integrated into PEBBs. In [4], use of IPT with multiple receivers and multiple separate transmitters is presented, however each transmitter requires an individual converter at its input.

Connecting transmitters in series is a preferable choice since it requires a single centralized converter instead of multiple converters at input stage and thus saves space inside the medium voltage converter. The IPT system described in [5] has multiple receivers and transmitters integrated into one module, however it needs additional voltage regulation stage after rectifier to get stable output voltage. Similarly, paper [6] requires clamping circuit against over voltage on each output to stabilize the output voltage.

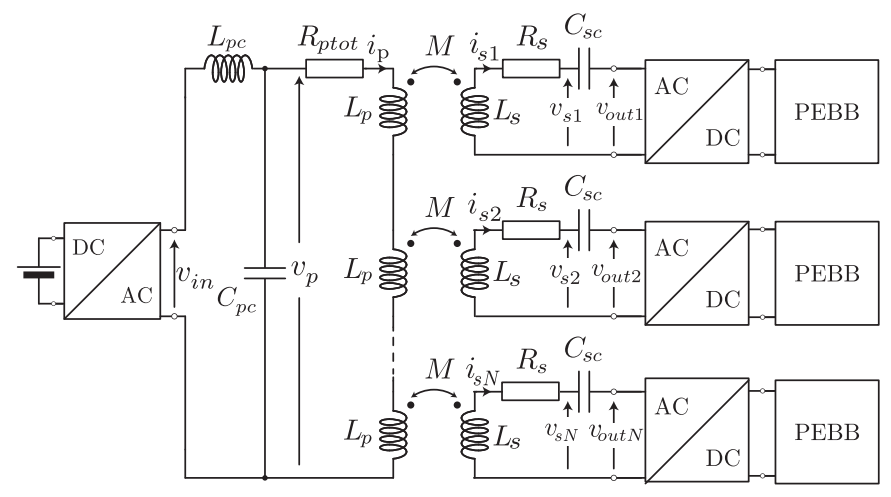

Fig. 1. LCL-S compensated IPT system with multiple receivers and series connected transmitters coils as APS for stacked PEBBs.

Unstable voltage output is caused by dependency among receivers. In order to achieve intrinsic stable voltage output, current source behavior is required on the transmitter side. Since adding compensation network is a common way to improve power transfer in IPT system [7], LCL compensation as shown in Fig.1 which behaves as a constant current source, provides benefits when supplying independent receivers. This compensation topology removes the dependence of output voltage from the load conditions and reduce the current stress of the bridge semiconductors [8].

Prior research on LCL has focused on the application with a single transmitter coil and optimization of compensation components [8]-[10]. However application of distributed transmitter coils are not discussed and optimization of operating parameters such as input voltage, switching frequency, converter topology, which can be chosen in a wide range, are ignored.

In this paper, the characteristic of APS of PEBBs is discussed, and based on it, the systematic design approach of the compensation network to achieve the optimization goal is proposed. Furthermore, the possible ranges of operating parameters are explored in order to determine their impact on the system efficiency. 
TABLE I

PARAMETER SPECIFICATIONS

\begin{tabular}{|c|c|c|}
\hline Design parameter & Variable & Range \\
\hline Output power of each receiver & $\mathrm{P}(\mathrm{W})$ & $10-150$ \\
\hline Operating frequency & $\mathrm{f}(\mathrm{kHz})$ & $50-500$ \\
\hline Total input DC voltage & $U_{\text {in }-D C}(\mathrm{~V})$ & 400 \\
\hline Output DC voltage of each receiver & $U_{\text {out }-D C}(\mathrm{~V})$ & $24 \pm 6$ \\
\hline Coupling factor & $\mathrm{k}$ & $0.02-0.25$ \\
\hline Amount of transmitters & $\mathrm{N}$ & 5 \\
\hline
\end{tabular}

\section{TECHNICAL REQUIREMENTS SPECIFICATIONS}

Typical medium voltage converter range is from $1 \mathrm{kVac}$ to 36kVac. Considering high insulation requirements between primary and secondary side coils, and low power levels indicating small coils sizes relative to distance between them, it is fair to assume that only low coupling factor $\mathrm{k}$ range can be achieved. Many modular converter topologies would have floating PEBBs and require relatively low amount of power to supply to each PEBB, e.g. from $10 \mathrm{~W}-150 \mathrm{~W}$ for sensors, gate drivers, etc. This is a very broad range of specification, that is hard to address with one unique solution. For those reason, this paper considers, without loss of generality, 5 stacked cells and $24 \mathrm{Vdc}$ as average output voltage of each receiver. Considering using 220Vac input and a PFC stage to supply DC voltage as input source, $400 \mathrm{~V}$ is chosen as input DC voltage. Since the coils are not designed yet, the winding resistance is assumed to be proportional to $a \sqrt{L}$, considering $\mathrm{L}$ being proportional to number of turns squared, while $a$ is additional scaling factor used for design evaluations (taken as 20 in this paper). Ranges of variables of interests are summarized in Tab. I.

Medium voltage converters may have redundant PEBBs, and if those PEBBs are not operating, the corresponding receiver is in no-load condition. In addition, loads in different PEBBs could be different and IPT should be robust against various conditions of loads.

In Fig. 1, $L_{p}$ and $L_{s}$ are transmitter (primary side) and receiver (secondary side) coil inductance respectively. $R_{\text {ptot }}=$ $N R_{p}$ is total equivalent winding resistance of multiple transmitter coils and $R_{s}$ is winding resistance on each receiver coil. $L_{p c}$ and $C_{p c}$ are compensation components on the transmitter side, and $C_{s c}$ is the compensation capacitor on the receiver side. The relations describing the system in Fig. 1 are:

$$
\begin{aligned}
V_{p} & =\left(R_{p t o t}+N j \omega L_{p}\right) I_{p}-\sum_{i=1}^{N} j \omega M I_{s i} \\
V_{s i} & =j \omega M I_{p}-\left(R_{s}+j \omega L_{s}\right) I_{s i}
\end{aligned}
$$

Where, $i \in[1, N]$. System (1) can be transferred into an equivalent circuit including current controlled voltage sources, as shown in Fig.2. The voltage imposed to the receivers side is controlled by the current of transmitter side. The impedance of the secondary reflected to the primary is $Z_{r}$ which will be elaborated in Section III. If the current going through the series connected transmitters could be kept constant, the different receivers will not influence each others' output voltage [11].

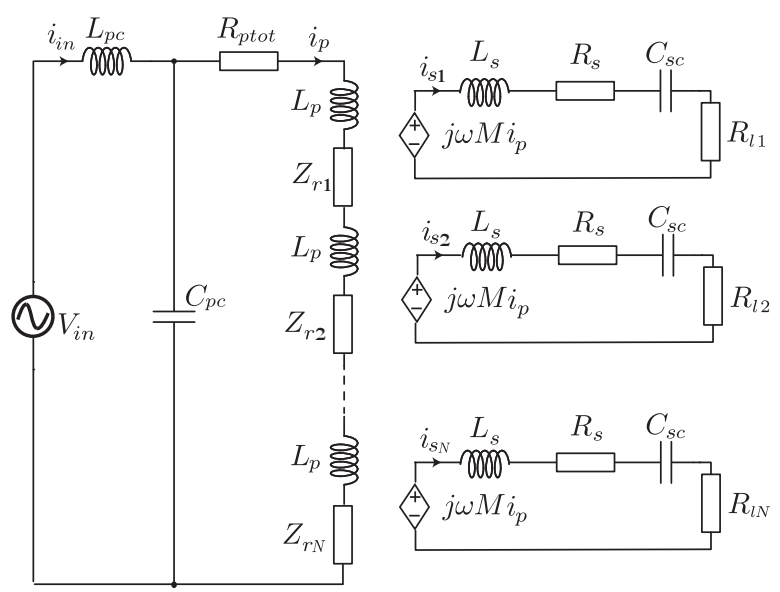

Fig. 2. Current source equivalent circuit of LCL-S circuit.

Since the receiver is implemented into PEBB, to achieve simplicity on the receiver side, only basic series compensation and parallel compensation are considered. With a constant voltage input at the receiver side, a parallel compensation network with an ideally compensated capacitor works as a constant current source, while a series compensation network works as a constant voltage source [12]. Therefore the series connected compensation capacitor $C_{s c}$ is deployed on the receiver side.

\section{DESIGN OPTIMIZATION}

In this section, selected topology will be further referred as LCL-S topology and the optimization design approach for series connected transmitter coils is illustrated. The operating range of interest is summarized in Tab. I. Following assumptions are made for simplicity: i) No loss on resonant tank and semiconductor switches; ii) Voltage source is modeled as a sinusoidal source (following first harmonic analysis principles and neglecting other harmonics); iii) Equivalent load $R_{l}$ is used to transfer the load from the DC side of half-bridge rectifiers to the AC side [13]:

$$
R_{l}=\frac{2}{\pi^{2}} \frac{U_{\text {out }-D C}^{2}}{P_{\text {out }}}
$$

\section{A. Maximum Efficiency}

Since the nominal required output power for each secondary side is fixed, the maximum efficiency instead of maximum output power becomes the optimization goal [14]. Link efficiency $\eta_{\text {link }}$ which is defined as the ratio of the power that reaches to the load to the total real power input to the link [7], is calculated and given in (3).

$Q_{p}$ and $Q_{s}$ are the quality factors of primary side and secondary sides respectively. $Q_{p}=\frac{w L_{p}}{R_{p}}$ and $Q_{s}=\frac{w L_{s}}{R_{s}}$. The $\alpha$ is the load factor defined as $\alpha=\frac{R_{l}}{w L_{s}}$, and $\omega_{n}$ is the normalized frequency defined as $\omega_{n}=\omega \sqrt{L_{s} C_{s c}}$.

The optimal operational frequency for series compensated 
$\eta_{l i n k}=\eta_{p} \eta_{s}=\frac{\sum_{i=1}^{N} \operatorname{Re}\left[Z_{r i}\right]}{\sum_{i=1}^{N} \operatorname{Re}\left[Z_{r i}\right]+R_{p t o t}} \frac{R_{l}}{R_{l}+R_{s}}=\frac{k^{2} \omega_{n}^{4} \alpha Q_{p} Q_{s}^{2}}{\omega_{n}^{4} \alpha Q_{s}\left(k^{2} Q_{p} Q_{s}+2\right)+k^{2} \omega_{n}^{4} Q_{p} Q_{s}+\omega_{n}^{4} \alpha^{2} Q_{s}^{2}+\omega_{n}^{4} Q_{s}^{2}-2 \omega_{n}^{2} Q_{s}^{2}+Q_{s}^{2}+\omega_{n}^{4}}$

secondary is determined by solving $\frac{\partial \eta_{l i n k}}{\partial \omega_{n}}=0$. The optimal $\omega_{n}$ is found to be 1 , therefore $\omega_{o p t}=\frac{\omega_{n} 1}{\sqrt{L_{s} C_{s c}}}$.

The efficiency at optimal frequency can thus be calculated:

$$
\eta_{l i n k}\left(\omega_{n}=1\right)=\frac{k^{2} Q_{p} \alpha}{\left(\alpha+k^{2} Q_{p}+\frac{1}{Q_{s}}\right)\left(\alpha+\frac{1}{Q_{s}}\right)}
$$

Based on the result of 4, with the same approach of calculating the optimal frequency, the optimal load factor is determined by solving $\frac{\partial \eta_{l i n k}\left(\omega_{n}=1\right)}{\partial \alpha}=0$. For similar and high coil quality factors $Q_{p}$ and $Q_{s}$, the optimal load factor is [13]:

$$
\alpha_{o p t-\eta}=\frac{\sqrt{1+k^{2} Q_{p} Q_{s}}}{Q_{s}} \approx k
$$

The maximum link efficiency under optimal frequency $\omega=$ $\frac{1}{\sqrt{L_{s} C_{s c}}}$ and optimal load factor $\alpha=k$ is:

$$
\eta_{\text {link-max }}=\frac{k^{3} Q_{p} Q_{s}}{\left(1+k Q_{s}+k^{2} Q_{p} Q_{s}\right)\left(1+k Q_{s}\right)}
$$

\section{B. Voltage Gain}

Reflected impedance $Z_{r i}$ of the $i^{\text {th }}$ receiver in Fig.2 is:

$$
Z_{r i}=\frac{\omega^{2} M^{2}}{R_{l i}+R_{s}+j \omega L_{s}+\frac{1}{j \omega C_{s} c}}
$$

With the aforementioned ideally compensated network at receiver side, the reflected impedance $Z_{r}$ becomes purely resistive and total reflected resistance is:

$$
R_{\text {rtot }}=\sum_{i=1}^{N} \frac{\omega^{2} M^{2}}{R_{l i}+R_{s}}
$$

By modeling total primary coil inductance using one inductor $L_{p t o t}=N L_{p}$, equivalent circuit of series connected transmitter coils is shown in Fig.3. The input impedance seen from the source side is:

$$
Z_{\text {in }}=Z_{1}+\frac{Z_{3}\left(Z_{2}+R\right)}{Z_{3}+\left(Z_{2}+R\right)}
$$

where, $Z_{1}, Z_{2}$ and $Z_{3}$ are purely inductive: $Z_{1}=j X_{1}=$ $j \omega L_{p c}, Z_{2}=j X_{2}=j \omega L_{p t o t}, Z_{3}=j X_{3}=\frac{1}{j \omega C_{p c}}$ and $R=$ $R_{\text {rtot }}+R_{\text {ptot }}$.

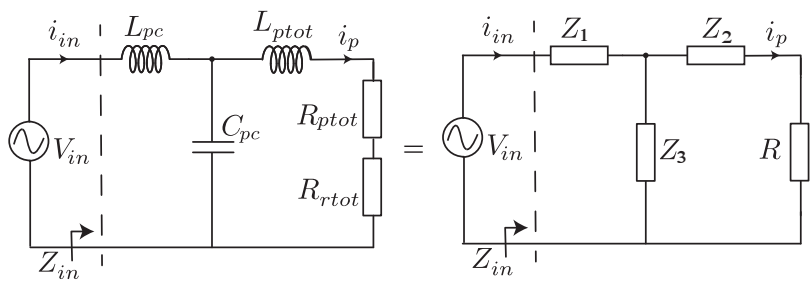

Fig. 3. Simplified equivalent circuit of series connected transmitters.
The current going through the primary coil is calculated with $Z_{\text {in }}$ :

$$
I_{p}=\frac{U_{\text {in }}}{Z_{\text {in }}} \frac{Z_{3}}{Z_{3}+\left(Z_{2}+R\right)}=\frac{U_{\text {in }}}{\left(R+Z_{2}\right)\left(1+\frac{Z_{1}}{Z_{3}}\right)+Z_{1}}
$$

Based on the analysis in Section II, the primary current going through each transmitter coil should remain constant, i.e. $I_{p}$ should be independent of $R$. Based on (10), the relation between $Z_{1}$ and $Z_{3}$ is determined:

$$
Z_{3}=-Z_{1} \Rightarrow C_{p c}=\frac{1}{\omega^{2} L_{p c}}
$$

The output voltage on $i^{\text {th }}$ receiver side with the assumption in (11) is derived as:

$$
V_{\text {out } i}=V_{\text {in }} \frac{k \sqrt{L_{p} L_{s}}}{L_{p c}} \frac{R_{l i}}{R_{l i}+R_{s}}
$$

One should notice that output voltage is independent of the load condition of other receivers. In (12), once the input DC voltage is fixed, the primary side AC voltage $V_{i n}$ depends on the inverter topology and phase angle. Primary and secondary coils as well as the primary compensation inductor will be designed to achieve desired voltage gain.

\section{Input Impedance Angle}

Substituting $Z$ with $j X$ in (9), and taking $Z_{3}=-Z_{1}$ into consideration:

$$
Z_{\text {in }}=\frac{X_{1}^{2} R}{R^{2}+\left(X_{1}-X_{2}\right)^{2}}+j \frac{X_{1}^{2}\left(X_{1}-X_{2}\right)}{R^{2}+\left(X_{1}-X_{2}\right)^{2}}
$$

The input impedance angle is:

$$
\tan (\theta)=\frac{\operatorname{Im}\left[Z_{i n}\right]}{\operatorname{Re}\left[Z_{i n}\right]}=\frac{X_{1}-X_{2}}{R}
$$

Desired input impedance can be achieved by adjusting $X_{2}$ and $X_{1}$. Defining the ratio of $X_{1}$ and $X_{2}$ as:

$$
\chi=\frac{X_{2}}{X_{1}}=\frac{L_{p t o t}}{L_{p c}}
$$

$\chi$ determines the input impedance angle. Under this relation, the output voltage is:

$$
V_{\text {out }}=U_{\text {in }} \frac{k \chi \sqrt{L_{s}}}{\sqrt{N L_{\text {ptot }}}} \frac{R_{l}}{R_{l}+R_{s}}
$$

A slightly inductive transmitter current is necessary for zero votlage switching (ZVS) conditions on the primary power stage, therefore $\chi$ slightly smaller than 1 is usually desired. $\chi_{\max }=1-\frac{\tan (\theta)_{\min } R}{X_{1}}$, with minimum impedance angle $\tan (\theta)_{\min }$ determined by input power stage semiconductor parameters. In addition, by setting $\chi=1$, purely resistive impedance can be achieved. 


\section{Design of the Compensation Network}

The design flow of compensation network is summarized based on discussion in previous sections and is shown in Fig. 4. The secondary resonant components values are firstly determined and then comes the primary resonant components. The coil design is out of scope of this paper.

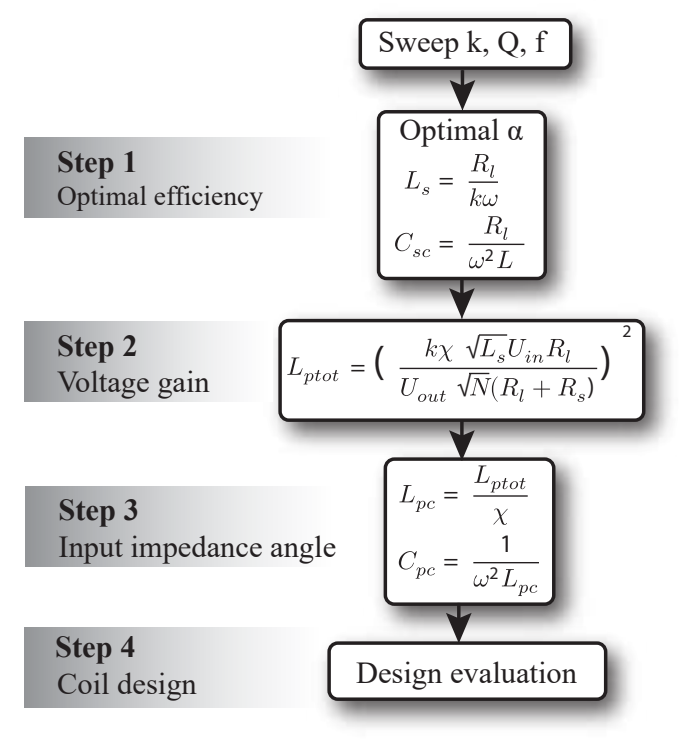

Fig. 4. Simplified design flow.

\section{DEPENDENCE OF THE OPERATING PARAMETERS}

In this section, the analysis has been done on the component and link efficiency dependence of different operating parameters including coupling factor $k$, switching frequency $f$, equivalent coil resistance as well as primary side inverter topology. In the following analysis, semiconductor losses are not considered and winding resistance is assumed as $20 \sqrt{L}$ without considering high frequency effects.

\section{A. Influence of $k, f$, and $U_{\text {in }}$}

According to the design approach presented in previous section, the values of achievable coupling factor $k$, and selected operating frequency $f$ will influence the ideal components value and the maximum efficiency.

With DC input voltage selected as $400 \mathrm{~V}$, both half-bridge and full-bridge inverters could be used for primary power stage, resulting in different $V_{i n}$ voltage. Assuming phase shift is $\pi$, the AC voltage amplitude are $\hat{U}_{F B}=\frac{4}{\pi} U_{D C}$, $\hat{U}_{H B}=\frac{2}{\pi} U_{D C}$.

The dependency of various components on inputs are plotted in Fig. 5 - Fig. 8, considering output power of $100 \mathrm{~W}$ at each receiver and parameters in Tab. I. The frequency is swept from $50 \mathrm{kHz}$ to $500 \mathrm{kHz}$ with the step of $22.5 \mathrm{kHz}$, the coupling factor is ranged from 0.05 to 0.25 with step of 0.02 and both $\mathrm{HB}$ and FB input stage inverter topologies are considered. According to Fig. 4, the values of coil inductance and compensation components are calculated. Based on (6), link efficiency is determined and plotted in Fig. 9.

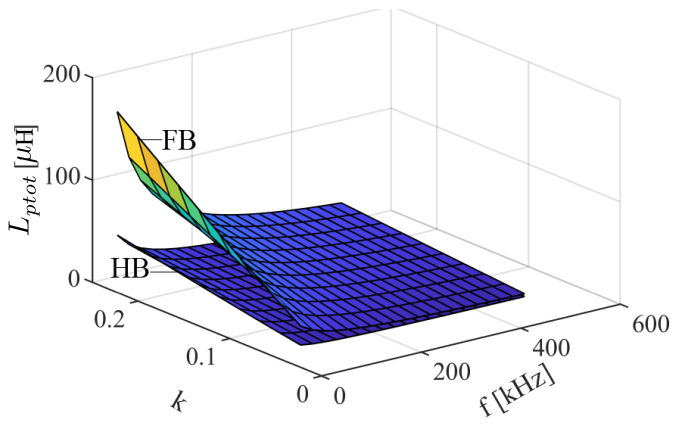

Fig. 5. Total coil inductance on the transmitter side as a function of $\mathrm{k}, \mathrm{f}$ and inverter topology under ideal compensation.

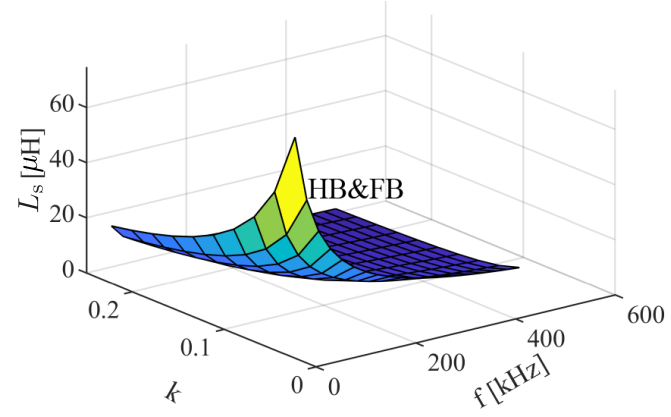

Fig. 6. Coil inductance on the receiver side as a function of $\mathrm{k}, \mathrm{f}$ and inverter topology under ideal compensation.

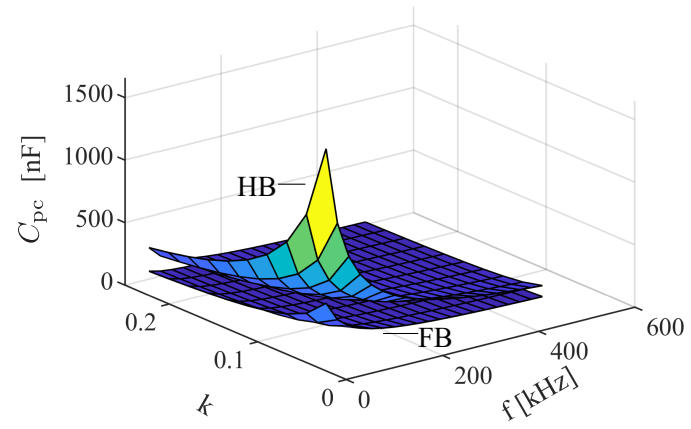

Fig. 7. Compensation capacitor on the transmitter side as a function of $k, f$ and inverter topology under ideal compensation.

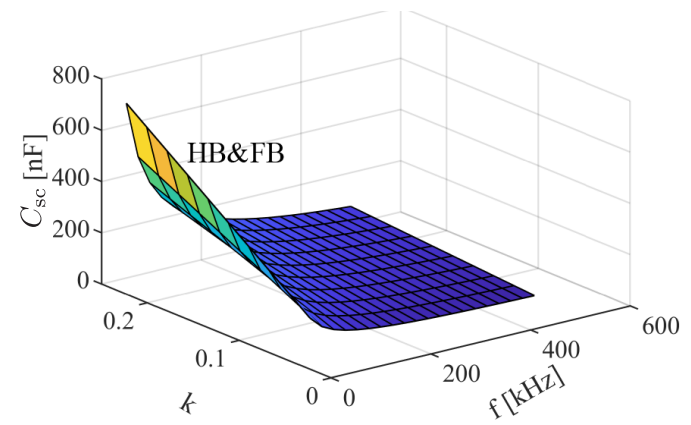

Fig. 8. Compensation capacitor on the receiver side as a function of $\mathrm{k}$, $\mathrm{f}$ and inverter topology under ideal compensation. 


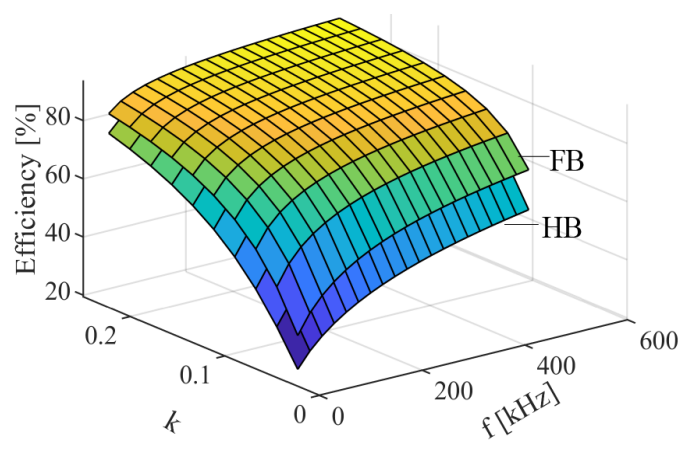

Fig. 9. Link efficiency as a function of $\mathrm{k}, \mathrm{f}$ and inverter topology under ideal compensation.

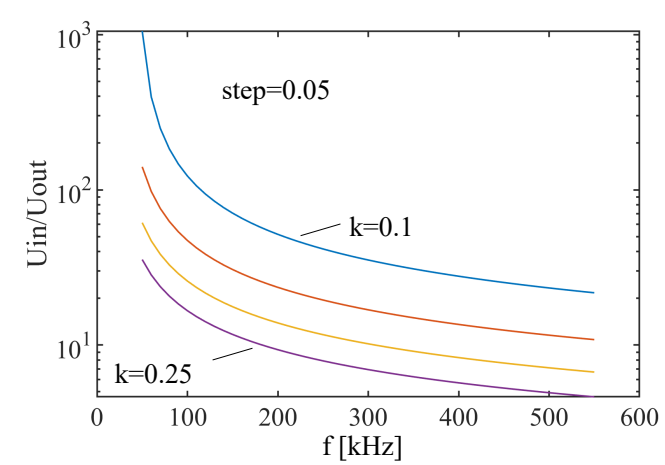

Fig. 10. Input voltage, coupling factor and frequency to achieve $90 \%$ efficiency.

As shown in the figures, the coupling factor and frequency will influence the design of both primary and secondary network, and the inverter topology only influence the design of the primary network. Higher frequency $f$ would lead to smaller component values and higher efficiency. It's also interesting to note that by using FB inverter smaller difference between transmitter and receiver inductance is achieved. Since $U_{\text {in }}$ would lead to bigger primary inductance value and thus bigger mutual inductance value, as consequence, it leads to higher link efficiency. Furthermore, $k, f$ and $U_{i n}$ should be as high as possible to get higher efficiency. In order to achieve certain efficiency, minimum $k, f$ as well as $\frac{U_{i n}}{U_{\text {out }}}$ can be calculated. As shown in Fig.10, each operating point on this plot is identical to $90 \%$ link efficiency. In order to attain $90 \%$ efficiency, if $k$ is small, higher switching frequency and higher input voltage needs to be implemented.

\section{B. Analysis of Equivalent Coil Resistance}

The link efficiency depends on the primary and secondary coil quality factors and coupling factor. Equivalent coil resistance $R_{p}$ and $R_{p}$ influence link efficiency by influencing quality factors. In Fig.11, the relation of $Q_{p}$ and $Q_{s}$ to achieve $90 \%$ efficiency for the optimized compensation network is shown. Once coil inductance is determined, minimum $Q_{p}$ and $Q_{s}$ determine maximum $R_{p}$ and $R_{s}$ which could be the guidance in coil design procedure. The design of coil is not covered in the paper.

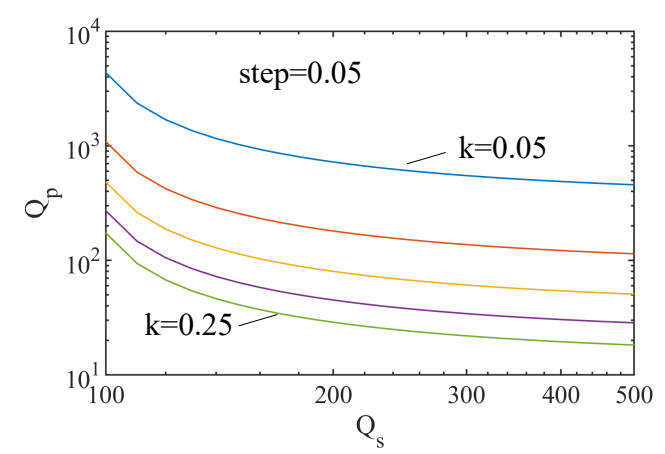

Fig. 11. Primary and secondary coil quality factors to achieve $90 \%$ efficiency.

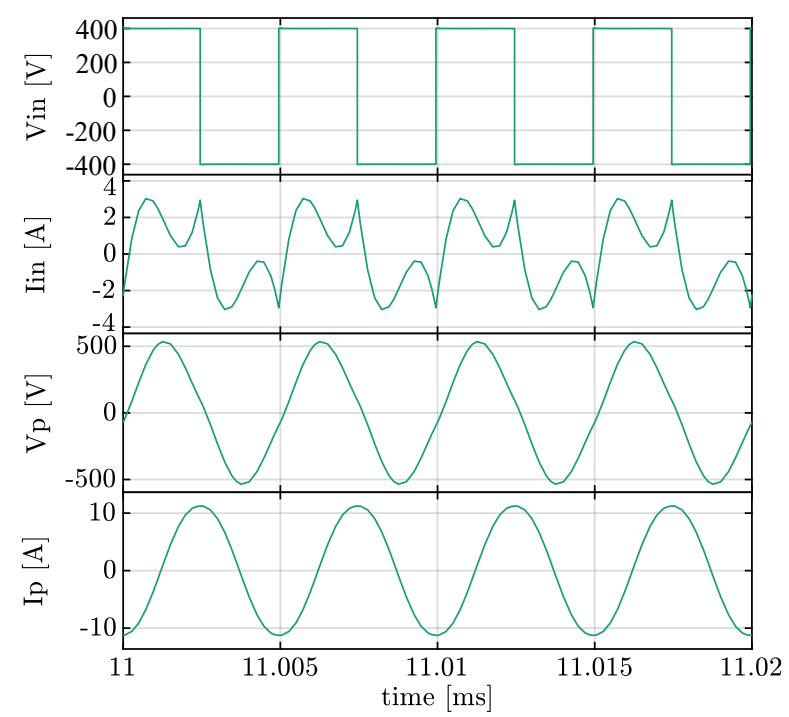

Fig. 12. From top to bottom: primary side input voltage before LCL network, current through primary compensated inductor, voltage across all the 5 primary coils and current through primary coils.

\section{Simulation Verification}

Simulation with PLECS is used to verify the results of the proposed design approach. Based on specifications in Tab. I, specifying switching frequency as $200 \mathrm{kHz}$, coupling factor as 0.2 , output power of each receiver as $100 \mathrm{~W}$ and using FB inverter at input stage and HB rectifier at output stage. Fig. 12 shows the voltage and current on primary side under full load condition before and after LCL compensation network.

A controlled current source instead of a resistive load is placed at each rectifier output to adjust the load condition. In order to see the influence of load change on secondary and primary side, this current source changes according to the test scenario presented in Fig. 13:

At $t_{0}$, all the load currents are set to 0 , simulation starts with primary working and all the secondaries are in no-load condition.

At $t_{1}$, all the load currents are set to rated currents.

At $t_{2}, 5^{\text {th }}$ load current is doubled.

At $t_{3}, 4^{\text {th }}$ load current is halved.

At $t_{4}, 3^{r d}$ and $4^{t h}$ load currents are set to 0 . 
At $t_{5}$, primary DC link voltage drops to $0.9 U_{D C}$.

Fig. 13 shows the DC output voltage of 5 receivers. Following can be concluded: i) The output of each receiver only depends on the load condition on its side and is independent of load conditions of other receivers. In the scenario, even though the $3^{r d}-5^{\text {th }}$ loads change, the output voltage of the $1^{\text {st }}$ and $2^{\text {nd }}$ receivers remain constant. ii) Load change of one receiver leads to a slight increase or decrease in this receiver's output voltage. During period $t_{2}-t_{5}$, the voltage of $5^{t h}$ receiver decreases $10 \%$ as its load doubles. During period $t_{3}-t_{4}$, the voltage of $4^{\text {th }}$ receiver increases $8 \%$ as its load is halved. iii) The drop of primary input voltage causes same drop on the output voltage. After $t_{5}$, the output voltage of each receiver drops $10 \%$ as the input voltage drops $10 \%$.

Fig. 14 shows the envelope of $\mathrm{AC}$ voltage before rectifiers and the envelope of AC currents through secondary side coils. $\mathrm{AC}$ voltage and current vary in the same pattern with the DC side voltage and current shown in 13. Therefore similar analysis could be made for Fig. 14.

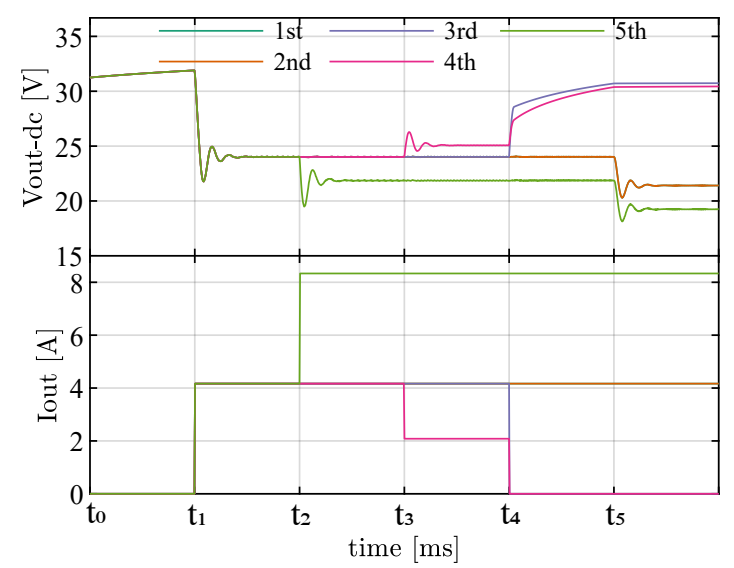

Fig. 13. Output DC voltage (image on top) and load current (image on bottom) of each receiver.

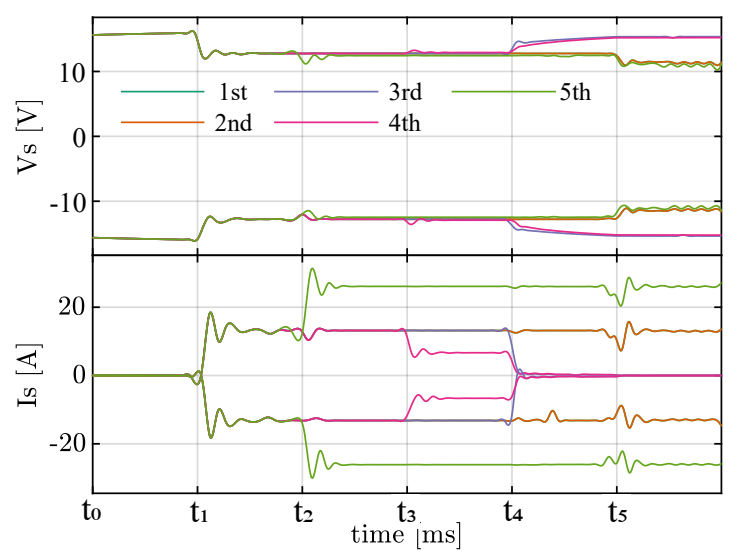

Fig. 14. Envelopes of AC output voltage (image on top) and current (image on bottom) of all 5 receivers before their rectifier stage.

\section{CONCLUSION}

To face the major concerns of APS for PEBBs, the parameter design approach of IPT system with series connected transmitter coils is proposed. The approach considers both optimal compensating components design and operating parameters selection to realize independent receivers, high efficiency, low reactive power simultaneously. By following the proposed design approach, each receiver performs robust output voltage characteristic against the load variation of other receivers.

\section{ACKNOWLEDGEMENT}

This research was supported by China Scholarship Council (NSCIS No. 201906280069)

\section{REFERENCES}

[1] R. Steiner, P. K. Steimer, F. Krismer, and J. W. Kolar, "Contactless energy transmission for an isolated $100 \mathrm{~W}$ gate driver supply of a medium voltage converter," in 2009 35th Annual Conference of IEEE Industrial Electronics, Nov. 2009, pp. 302-307.

[2] B. Wunsch, D. Zhelev, and B. Oedegard, "Externally-fed auxiliary power supply of MMC converter cells," in 2016 18th European Conference on Power Electronics and Applications (EPE'16 ECCE Europe). Karlsruhe: IEEE, Sep. 2016, pp. 1-10.

[3] C. Marxgut, J. Biela, J. W. Kolar, R. Steiner, and P. K. Steimer, "DC-DC converter for gate power supplies with an optimal air transformer," in 2010 Twenty-Fifth Annual IEEE Applied Power Electronics Conference and Exposition (APEC). Palm Springs, CA: IEEE, Feb. 2010, pp. 1865-1870.

[4] B. Wunsch, J. Bradshaw, I. Stevanovic, F. Canales, W. Van-der-Merwe, and D. Cottet, "Inductive power transfer for auxiliary power of medium voltage converters," in 2015 IEEE Applied Power Electronics Conference and Exposition (APEC). Charlotte, NC, USA: IEEE, Mar. 2015, pp. 2551-2556.

[5] J. Afsharian, B. Wu, and N. Zargari, "High-voltage isoltated multiple outputs DC/DC power supply for GCT gate drivers in medium voltage (MV) applications," in 2013 28th Annual IEEE Applied Power Electronics Conference and Exposition (APEC), Mar. 2013, pp. 1480-1484.

[6] D. Peftitsis, M. Antivachis, and J. Biela, "Auxiliary power supply for medium-voltage modular multilevel converters," in 2015 17th European Conference on Power Electronics and Applications (EPE'15 ECCEEurope). Geneva: IEEE, Sep. 2015, pp. 1-11.

[7] K. van Schuylenbergh and R. Puers, Inductive Powering: Basic Theory and Application to Biomedical Systems, ser. Analog Circuits and Signal Processing. Springer Netherlands, 2009.

[8] B. Esteban, M. Sid-Ahmed, and N. C. Kar, "A Comparative Study of Power Supply Architectures in Wireless EV Charging Systems," IEEE Trans. Power Electron., vol. 30, no. 11, pp. 6408-6422, Nov. 2015.

[9] H. Feng, T. Cai, S. Duan, J. Zhao, X. Zhang, and C. Chen, "An LCCCompensated Resonant Converter Optimized for Robust Reaction to Large Coupling Variation in Dynamic Wireless Power Transfer," IEEE Trans. Ind. Electron., vol. 63, no. 10, pp. 6591-6601, Oct. 2016.

[10] N. A. Keeling, G. A. Covic, and J. T. Boys, "A Unity-Power-Factor IPT Pickup for High-Power Applications," IEEE Trans. Ind. Electron., vol. 57, no. 2, pp. 744-751, Feb. 2010.

[11] H. Hao, G. A. Covic, and J. T. Boys, "An Approximate Dynamic Model of LCL-T-Based Inductive Power Transfer Power Supplies," IEEE Trans. Power Electron., vol. 29, no. 10, pp. 5554-5567, Oct. 2014.

[12] O. Stielau and G. Covic, "Design of loosely coupled inductive power transfer systems," in PowerCon 2000. 2000 International Conference on Power System Technology. Proceedings (Cat. No.00EX409), vol. 1, Dec. 2000, pp. 85-90 vol.1.

[13] R. Bosshard, J. W. Kolar, J. Mühlethaler, I. Stevanović, B. Wunsch, and F. Canales, "Modeling and $\eta-\alpha$-Pareto Optimization of Inductive Power Transfer Coils for Electric Vehicles," IEEE Trans. Emerg. Sel. Topics Power Electron., vol. 3, no. 1, pp. 50-64, Mar. 2015.

[14] S. Y. R. Hui, W. Zhong, and C. K. Lee, "A Critical Review of Recent Progress in Mid-Range Wireless Power Transfer," IEEE Trans. Power Electron., vol. 29, no. 9, pp. 4500-4511, Sep. 2014. 